\title{
Modelo conceptual de entornos geográficos dinámicos
}

\section{Conceptual Model of Dynamic Geographic Environment}

\author{
Martínez-Rosales Miguel Alejandro \\ Laboratorio de Procesamiento Inteligente de Información \\ Geo-espacial \\ Centro de Investigación en Computación \\ Instituto Politécnico Nacional \\ Correo:mamartinezr@ipn.mx
}

\author{
Levachkine Serguei \\ Laboratorio de Procesamiento Inteligente de Información \\ Geo-espacial \\ Centro de Investigación en Computación \\ Instituto Politécnico Nacional \\ Correo:sergei@cic.ipn.mx
}

Información del artículo: recibido: marzo de 2011, reevaluado: septiembre de 2012, aceptado: junio de 2013

\section{Resumen}

En los entornos geográficos hay muchos y diferentes tipos de entidades geográficas, como automóviles, árboles, personas, edificios, tormentas, huracanes, etcétera. Estas entidades se clasifican en dos grandes grupos: objetos geográficos y fenómenos geográficos. Por su naturaleza, un entorno geográfico es dinámico, por lo que el modelado de forma estática no es suficiente. $\mathrm{Al}$ considerar que los entornos geográficos son dinámicos aparece un nuevo tipo de entidad llamado evento. El propósito de realizar un modelado de este tipo, el cual considere eventos, se debe a que las relaciones semánticas que se pueden obtener son más ricas que en modelos que no lo hacen. En este artículo se presenta la conceptualización de este modelo, el cual está basado en tratar a cada entidad como una sola y por medio de la unión de las historias de cada una de ellas y relaciones entre estas, formar la descripción de todo un entorno geográfico dinámico completo. El principal objetivo es el poder modelar sistemas a nivel conceptual que hagan uso de información espacial y temporal, para que posteriormente sirva como el motor semántico para dichos sistemas.

\section{Descriptores:}

- eventos

- modelado dinámico

- GIS

- espacio-temporal

- ontología semántica 


\begin{abstract}
In geographic environments, there are many and different types of geographic entities such as automobiles, trees, persons, buildings, storms, hurricanes, etc. These entities can be classified into two groups: geographic objects and geographic phenomena. By its nature, a geographic environment is dynamic, thus, it's static modeling is not sufficient. Considering the dynamics of geographic environment, a new type of geographic entity called event is introduced. The primary target is a modeling of geographic environment as an event sequence, because in this case the semantic relations are much richer than in the case of static modeling. In this work, the conceptualization of this model is proposed. It is based on the idea to process each entity apart instead of processing the environment as a whole. After that, the so called history of each entity and its spatial relations to other entities are defined to describe the whole environment. The main goal is to model systems at a conceptual level that make use of spatial and temporal information, so that later it can serve as the semantic engine for such systems.
\end{abstract}

\section{Introducción}

En la actualidad, las ciencias de información geográfica se enfocan en capturar el dinamismo de entornos geográficos y describir la semántica de las entidades geográficas (objetos y eventos) y las relaciones espaciales entre estas a través del tiempo. En el mundo existe un gran número de entidades geográficas, las cuales por naturaleza no son estáticas, es decir, su comportamiento es dinámico dentro de un entorno geográfico (Langran, 1972). Por esta razón, los esfuerzos de la comunidad se enfocan en el desarrollo de modelos basados en eventos, en donde estos son la parte principal del modelo (Worboys y Hornsby, 2004). La dinámica se refiere a los sucesos en el mundo que envuelven cambios o movimiento. Derrames de petróleo, tornados o manifestaciones son todos ejemplos de la dinámica geográfica.

Hasta la fecha, la mayoría de los esfuerzos en el modelado de datos se ha enfocado en trabajar con snapshots estáticos que reducen la profundidad natural de la dinámica del mundo real para simplificarla, abstrayendo perspectivas que son fijas o estáticas en alguna forma. Para dirigirse hacia este camino, es necesario responder algunas preguntas clave: ¿qué tipos de fenómenos geográficos son fundamentalmente dinámicos?, ¿qué hacen los datos geográficos dinámicos?

Cuando se lleva a cabo una descripción explícita de un entorno específico, esta representa las propiedades y relaciones espaciales entre los objetos que pertenecen al entorno geográfico. Estas propiedades y relaciones sólo describen el estado del entorno cuando la descripción se realiza, pero no representan explícitamente los cambios que ocurren a través del tiempo. Por esta razón no es posible inferir automáticamente las cosas que ocurren cuando hay cambios entre descripciones sucesivas.

Ahora bien, no es suficiente analizar lo que ocurre entre descripciones sucesivas, porque de esta forma todos los eventos se tratan como si tuvieran la misma temporalidad, lo cual no ocurre en la realidad, por lo que esta semántica asociada se pierde.

En este artículo se presenta la conceptualización para el modelado de un entorno geográfico dinámico, el cual está basado en obtener la historia desde la perspectiva de cada entidad geográfica (una historia de entidades significan todos los cambios sucesivos en relaciones espaciales y las propiedades que tuvo esa entidad). Luego, por medio de la unión de estas historias y sus relaciones, se genera la descripción del entorno geográfico buscando para obtener un mayor contenido semántico al expresar explícitamente, tanto las historias como sus partes más importantes al estar relacionadas con otras. El principal objetivo de esta propuesta de modelado es mejorar las representaciones actuales que sólo consideran aspectos geográficos, dejando los aspectos temporales a la interpretación del analista de la información, dando como resultado respuestas ambiguas o lentas. Por otro lado, si se desarrollan sistemas basados en la conceptualización que aquí se propone, se mejora la calidad y el tiempo de respuesta del sistema, ya que estará basada en una ontología espacio-temporal, por lo que el modelo se puede utilizar para el desarrollo de sistemas automatizados en la toma de decisiones que requieran una respuesta rápida, además de que tengan la cualidad de analizar la información a nivel semántico.

El artículo está organizado de la siguiente manera. La siguiente sección contiene un recorrido por el estado 
del arte relacionado con el tema de la presente investigación. Después se presenta la metodología y más adelante los resultados obtenidos con dicha metodología. Por último se exponen las conclusiones y se dan algunas sugerencias para el trabajo futuro.

\section{Estado del arte}

Desde que se reconoce que los entornos geográficos son dinámicos y que su inclusión es necesaria, la siguiente generación de sistemas de información geográfica debe incorporar y manejar de forma nativa el tiempo. A pesar de esto, aún no existe un consenso en la forma de cómo se debe llevar a cabo. Hasta el momento, hay dos vertientes de cómo realizar el modelado de escenarios geográficos; estas son: el enfoque endurantista y perdurantista, que se refieren a cómo es la persistencia de los objetos a través del tiempo. El primer enfoque sostiene que los objetos están en tres dimensiones, solo tienen partes espaciales y persisten totalmente en todo momento de su existencia. El segundo, sostiene que los objetos están en cuatro dimensiones, tienen partes temporales y sólo persisten parcialmente en cada momento de su existencia. La diferencia entre los dos enfoques se trata en los trabajos de (Galton y Worboys, 2004).

Sin movimiento o cambio, no hay forma de detectar o medir la dinámica en un entorno geográfico. La percepción del cambio implica dos hipótesis. Primero, lo que se observa sigue siendo el mismo objeto desde un tiempo $t_{1}$ a otro $t_{2}$. Las identidades permiten al observador reconocer y trazar los mismos objetos para el análisis del cambio. En Hornsby y Egenhofer (2000) se propone un modelo de cambio basado en la identidad con un conjunto de primitivas y operaciones. El conjunto de primitivas incluye estados de identidad de existencia y no-existencia. Las ligas de transición entre los estados de identidad de los objetos, expresan el cambio en el objeto pasando de un estado a otro. Sin embargo, el modelo tiene dos clasificaciones: con historia y sin historia, para existencia y no-existencia, respectivamente. Las operaciones pueden entonces usarse para determinar los tipos de cambio sobre uno de los cuatro posibles estados de identidad a otro.

Un método común para capturar cambios es trabajar con una secuencia de snapshots o muestras discretas en momentos secuenciales de tiempo (Langran, 1972; Peuquet, 1994). El enfoque de snapshot es un procedimiento usado por cartógrafos basado en secuencias animadas de mapas (DiBiase et al., 1992). Se pueden aplicar varias técnicas, por ejemplo, jugar con la secuencia de muestras discretas a diferentes velocidades como los cuadros de una película, cambiando la dura- ción de una escena para afectar el ritmo de una animación, o alterar el orden en que se presentan las escenas. Investigadores interesados en capturar la complejidad de los procesos subyacentes, están a menudo insatisfechos con el enfoque de snapshot, porque este método pasa por alto los eventos, cada uno de ellos ocurre por separado y tiene lugar entre los snapshots (Chrisman, 1998). Los cambios que ocurren entre los snapshots no se almacenan explícitamente, deben determinarse comparando los patrones espaciales de dos estados sucesivos, otra desventaja comúnmente citada de los snapshots es el almacenamiento de información redundante (Langran,1972; Peuquet y Wentz, 1994) que ocurre desde la representación de localizaciones donde no hay cambio alguno.

En el trabajo de (Galton, 2003), se identifican los conceptos generales necesarios para soportar un modelo orientado a eventos y procesos para sistemas de información geográficos dinámicos. Se introducen de manera informal los conceptos de objeto, estado, proceso y evento, pero sin llegar a un consenso de cómo deben definirse formalmente. Galton sostiene que los objetos y procesos tienen la posibilidad de tener cambios, sin embargo difieren significativamente con respecto a los eventos, pues los primeros tienen propiedades que pueden o no depender del tiempo, mientras los segundos tienen únicamente propiedades que dependen del tiempo. Por otro lado, sostiene que los objetos y procesos pertenecen a un snapshot del mundo real, mientras que los eventos pertenecen a un registro histórico, en el sentido de que se agregan en el transcurrir del tiempo.

En (Hornsby y Cole, 2007) se presentan los sucesos dinámicos u ocurrencias experimentadas por entidades en un dominio geoespacial, donde se modelan como eventos a partir de un análisis, muestran cómo la información significativa acerca del movimiento de objetos puede ser abstraída. Presentan un enfoque para abstraer patrones de movimiento a partir de secuencias de eventos basadas en atributos clave: identidad del objeto, descripción del evento y localización del evento. Sin embargo, adoptaron la perspectiva común de bases de datos, donde los eventos se modelan como instantáneos, es decir, eventos que no tienen duración y marcan un cambio en el estado de un objeto. Por lo que no consideran el caso en que los eventos tengan una cierta duración y, por lo tanto, las relaciones que pueden existir entre éstos no se consideran.

En Campos y Hornsby (2004) se presenta un modelado de datos para eventos geográficos cíclicos (los que son repetitivos con respecto al tiempo). En el artículo se introduce una nueva estructura que captura las posi- 
bles relaciones entre ocurrencias de eventos cíclicos, así como la frecuencia en que cada relación temporal ocurre. Se enfocan en las características temporales de eventos cíclicos y tratan las relaciones entre eventos que pueden expresarse en términos de restricciones temporales. Como sólo tratan eventos que ocurren repetidamente de manera cíclica, no tratan eventos acíclicos (los que surgen esporádicamente), los cuales también deben considerarse para un modelado mejor y más completo de los sucesos que ocurren en el mundo real.

Tradicionalmente, los sistemas de información geográfica se desarrollan desde enfoques estáticos, que usualmente están fundamentados en el paradigma basado en objetos, donde el mundo se percibe como una colección de entidades con propiedades que incluyen extensión espacial o en un paradigma basado en campos, donde los campos son atributos que varían espacialmente. Adicionalmente, se sabe que la siguiente generación de los sistemas de información geográfica requiere incorporar el tiempo de forma explícita (Langran, 1972; Worboys, 1994; Peuquet, 1994, 2001, 2002; Raper, 2000). A pesar de esto, aún no hay un consenso de lo que esto significa o cómo llevarlo a cabo.

La idea que se ha presentado en los trabajos previos es extender los enfoques basados en campos y objetos para permitir la inclusión de cambios en su comportamiento. Para esto, es necesario generalizar estos enfoques, además de explorar las diferentes formas en que pueden combinarse y dirigirse a un enfoque endurantista o perdurantista (Hales y Johnson, 2003). El presente trabajo propone el concepto de historia de una entidad geográfica, el cual permite combinar los dos enfoques tradicionales y quitar su dependencia del origen de los datos.

\section{Metodología}

Cada historia cuenta el comportamiento que tuvo la entidad geográfica durante el periodo en el que se describe el entorno. En un principio las historias parecen estar disjuntas unas de otras al tratar a cada entidad de forma individual. Pero dentro del entorno geográfico las entidades están relacionadas unas con otras por medio de relaciones espaciales, por lo que las historias que resultan también están relacionadas. Estas historias, conjuntamente con las relaciones entre éstas, son las que forman la descripción del entorno geográfico completo.

De acuerdo con esto, se propone la ecuación (1) que tiene como objetivo describir el comportamiento dinámico de un entorno geográfico dinámico dentro de un periodo que se denota por $T$.
$\operatorname{Description}($ Environment $(T))=\bigcup_{i=0}^{n} h i s t\left(\right.$ ent $\left._{i}\right)$

donde:

Environment ( ) representa un entorno geográfico con todas las entidades que pertenecen a él. Podemos verlo como un conjunto de objetos geográficos, los cuales están relacionados entre sí.

Description ( ) es una función que se encarga de obtener la descripción en términos temporales de un entorno geográfico. Esta relaciona las historias de las entidades para formar la historia completa del entorno $\mathrm{y}$, por ende, su descripción.

hist $\left(\right.$ ent $_{i}$ ) representa la historia de una entidad geográfica $e n t_{i}$ durante el periodo $T$.

Esta descripción está compuesta por las historias que cada entidad tiene en el entorno durante su tiempo de vida (existencia) en el intervalo de tiempo T. Las historias de todas las entidades del entorno geográfico están relacionadas (en la ecuación (1) se denota como una unión) para formar la descripción de todo el entorno.

Se denota como EG al conjunto de entidades geográficas que pertenecen al entorno geográfico de interés a describir. EG es un subconjunto de entidades del mundo real y ent ${ }_{i}$ es una instancia para cada una de las entidades geográficas, por lo tanto, ent $t_{i} \in G$.

El número de entidades geográficas que participan en la descripción del $E G$ en el periodo $T$ debe ser finito, por lo tanto, el número total de entidades está dado por $n$.

En la ecuación (2) se define la función hist ( ), la cual está conformada por la unión de todos los cambios que una entidad geográfica en específico ha sufrido durante el intervalo de tiempo $T$ en que se realiza la descripción del entorno geográfico.

$\operatorname{hist}\left(e n t_{i}\right)=\bigcup_{l=1}^{m} C C_{l}\left(e n t_{i}, t_{j}, t_{k}\right)$

Los cambios que ocurren se identifican por las cadenas de cambio que representan explícitamente los cambios para cada entidad geográfica, ya sea en sus propiedades y/o en sus relaciones espaciales. La ecuación (3) muestra la definición de las cadenas de cambio, denotadas con la función CC( ).

$$
\begin{aligned}
& C_{l}\left(e n t_{i}, t_{j}, t_{k}\right)= \\
& \bigcup_{\forall t_{j} \in T \wedge \forall t_{k} \in T \mid t_{j}<t_{k}}\left[\operatorname{desc}\left(e n t_{i}, t_{j}\right) \operatorname{\Phi desc}\left(e n t_{i}, t_{k}\right)\right]
\end{aligned}
$$

donde: 
desc $\left(e n t_{i}, t_{j}\right)$ y desc $\left(e n t_{i}, t_{k}\right)$ representan las propiedades que tiene la ent $t_{i}$ en los instantes $t_{j}$ y $t_{k^{\prime}}$, respectivamente. Estas descripciones pueden ser tanto en aspectos espaciales como en temporales.

El operador $\Phi$ representa el cambio identificado entre las descripciones en los instantes $t_{j}$ y $t_{k}$. Es decir, de acuerdo con la ecuación (4), la cual representa la descripción de una entidad en un momento dado, el cambio puede ocurrir en tres partes principales: cambio en el valor de una propiedad, cambio de relación espacial con otras entidades y cambio de relación temporal con los eventos existentes en el momento que se realiza la descripción.

Entonces, cuando ocurre un cambio en alguna de las propiedades, se puede identificar porque el valor de esa propiedad en $t_{j}$ es diferente en $t_{k}$, es decir, este $a u$ mentó, disminuyó o simplemente cambió. Esta comparación se debe realizar para cada una de las propiedades de la entidad geográfica.

Para el caso de cambio en las relaciones espaciales (tabla 1) están dadas en por las siguientes categorías:

- Relaciones topológicas, son invariantes a transformaciones geométricas (Egenhofer, 1989; Egenhoferet y Herring, 1990); por ejemplo, "la calle cruza las vías del tren".

- Relaciones métricas, están dadas en términos de distancia y direcciones (Peuquet y Xiang., 1987); por ejemplo, "la montaña está al norte".
- Relaciones de orden, pueden ser de orden parcial o total (Kainz, 1989), como las descritas por preposiciones tales como enfrente de, detrás de, sobre y antes de (Freeman, 1975; Chang et al., 1989; Hernández, 1991); por ejemplo, "el automóvil está enfrente de la casa".

Por ejemplo, si en $t_{j}$ una persona está dentro de-el salón y en $t_{k}$ esta misma persona ahora está fuera del salón, entonces podemos decir que: la persona salió del salón, en este caso $\Phi$ se sustituye por salió. De esta manera, si se tienen $n=6$ relaciones topológicas, se tienen 36 posibles valores para $\Phi$. Esto mismo sucede con las relaciones métricas, la cuales $n=10$ teniendo 100 posibles valores para $\Phi$. Finalmente, para las relaciones de orden parcial donde $n=16$, se tienen 256 valores posibles para $\Phi$.

Si el cambio ocurre en alguna relación temporal de la entidad geográfica con algún evento (las relaciones temporales se dan en la tabla 2), el cambio identificado puede ser, por ejemplo, si la entidad está en una clase de matemáticas, la relación es: "Juan está dentro de clase de matemáticas", pero si en un tiempo posterior ya no está dentro de esa clase, significa que primero participaba en ese evento y después ya no. Entonces podemos decir que el cambio identificado es "salió" de su clase. Por lo tanto, de igual manera que con las relaciones espaciales, si tenemos cuatro relaciones temporales, entonces podemos llegar a tener 16 posibles cambios que se pueden identificar.
Relación espacial

Descripción
Tabla1. Relaciones espaciales entre objetos geográficos
Topológicas
Las siguientes relaciones topológicas que se consideran son las dadas en Martinez (2006): Conecta, Comparte, Comparte_límite, Dentro, Interseca y Cruza.

Métricas

De orden
Se consideran los valores de dirección: Norte $(\mathrm{N})$, Sur $(\mathrm{S})$, Este (E), Oeste (O), Noreste (NE), SurEste (SE), NorOeste (NO), SurOeste (SO), NorNorEste (NNE), EsteNorEste (ENE), SurSurEste (SSE), EsteSurEste (ESE), SurSurOeste (SSO), OesteSurOeste (OSO), OesteNorOeste (ONO) yNorNorOeste (NNO). Junto a un valor de distancia. Por ejemplo: (N, 5km), (SE, $2.3 \mathrm{~km})$.

Para este caso se toman en cuenta las siguientes relaciones: enfrente de, detrás de, sobre, debajo de, antes de, después de, junto a, al lado de, a la izquierda y a la derecha de. 


\begin{tabular}{ll}
\hline \multicolumn{1}{c}{ Relación } & \multicolumn{1}{c}{ Descripción } \\
\hline Inicia & El objeto geográfico causa el inicio de un evento. \\
Termina & El objeto geográfico causa que el evento termine. \\
Participa & $\begin{array}{l}\text { El objeto geográfico pertenece al evento, es decir, el objeto } \\
\text { comparte la misma localización con el evento en el momento que } \\
\text { se desarrolla ese evento. }\end{array}$ \\
& El objeto geográfico no comparte nada con el evento. \\
\hline
\end{tabular}

Tabla 2. Relaciones temporales entre entidades geográficas y eventos
Cabe señalar que los instantes de tiempo en los que se obtiene la descripción de una entidad geográfica ent $t_{i}$ es diferente para cualquier otra entidad. Esto quiere decir que dependiendo de la naturaleza tanto espacial y temporal de cada entidad, se eligen los instantes en el tiempo con el objetivo de capturar la mayor cantidad de información semántica.

En la ecuación (3) se usan descripciones en un instante para una entidad geográfica. El resultado de obtener la descripción de una entidad ent $t_{i}$ en un instante $t_{t}$ se realiza por medio de la función $\operatorname{desc}($ ) y depende tanto del contexto general, como de un contexto local para cada entidad geográfica. Primero, en la ecuación (4) se define la función $\operatorname{desc}($ ).

$\operatorname{desc}\left(\right.$ ent $\left._{i}, t_{k}\right)=\left\{\left\{p_{p} \theta\right.\right.$ Valor $\},\left\{\right.$ ent $_{i}$ went $\left._{j}\right\},\left\{\right.$ ent $\left.\left._{i} \omega e_{e}\right\}\right\}$

Donde $p_{p} \in P$ y $P=\left\{p_{1}, p_{2}, \ldots, p_{n}\right\}$ representa el conjunto de propiedades de ent $t_{i}$ y $\theta$ representa un operador del siguiente conjunto $\{<, \leq,=, \neq, \geq,>\}$. La relación espacial que existe entre $e n t_{i}$ y alguna otra entidad geográfica ent $t_{\text {. que pertenezca al contexto local }}{ }^{1}$ de ent $t_{i}$ está representada por $\psi$, el cual puede tomar como valor alguna de las relaciones espaciales (tabla 1). Las relaciones temporales representadas por $\omega$ son algunas relaciones temporales de la tabla 2 entre $e n t_{i}$ y un evento $e_{e}$. Estos $e_{e} \in E=\left\{e_{1}, e_{2}, \ldots, e_{m}\right\}$, donde $E$ representa el conjunto de eventos que se desarrollan en el periodo $T$.

\section{Relaciones con eventos}

En la figura 1 se muestra un diagrama de cómo se clasifican los eventos y cómo se relacionan con otros eventos y con entidades geográficas. Se definen tres relaciones axiomáticas $\omega=\{$ inicia, modifica, finaliza $\}$, las cuales son

1 Contexto local. Define a las entidades que son de interés ent. para una entidad en particular $e n t_{i}$, con $i \neq j$ y $e n t_{j} \subseteq E$. Su finalidad es excluir aquellas entidades que no son de interés para ent $_{i}$, con lo cual se reduce el espacio de búsqueda representando únicamente las relaciones más importantes o representativas para cada una de las entidades geográficas.

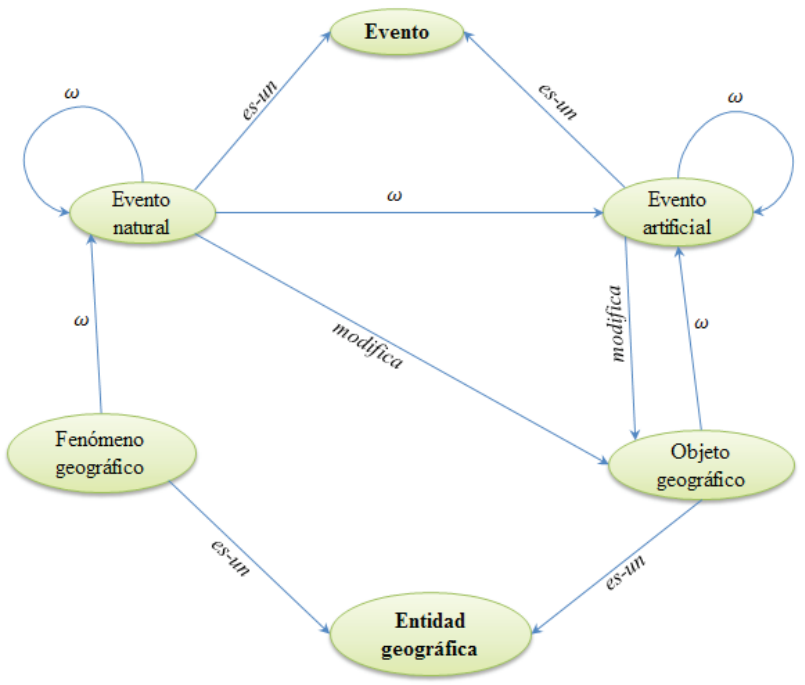

Figura 1. Conceptualización de eventos y sus relaciones

suficientes para poder relacionar evento/evento, evento/ objeto y objeto/evento.

En la figura 1 se puede observar que un evento natural puede iniciar, modificar o finalizar otro evento natural. Por ejemplo, si está lloviendo fuertemente, genera que un río se desborde. También podemos ver que un evento natural inicia, modifica o finaliza un evento artificial, por ejemplo, si está nevando, los vehículos en esta carretera disminuyen la velocidad.

Ahora bien, los eventos artificiales también inician, modifican of finalizan otro evento artificial, por ejemplo, si vamos manejando y pisamos el freno, esto causa que disminuya la velocidad del vehículo. En este caso, el evento de pisar el pedal del freno inicia el evento de disminuir la velocidad del vehículo. Un evento artificial no puede iniciar, modificar o finalizar un evento natural, es decir, no podemos generar lluvia, tampoco que caiga nieve, iniciar un tornado o una tormenta.

Un evento natural sólo puede modificar un objeto geográfico, pero no iniciarlo o finalizarlo. Por ejemplo, una lluvia muy fuerte causa que se modifique el caudal del río, es decir, únicamente se modifican las propiedades del río, causado por el evento de la lluvia. 
Por otra parte, los fenómenos geográficos pueden iniciar, modificar y finalizar un evento natural, por ejemplo, un huracán indica que comience a llover, hacer mucho viento o modificar la temperatura ambiente.

Desde el punto de vista de los objetos geográficos, estos tienen una relación más "estrecha con los eventos". Primero, un objeto geográfico, sin importar si es fiat o bonafide, puede iniciar, modificar o finalizar un evento artificial. Por ejemplo, un auto genera eventos como moverse o frenar. Tanto los eventos naturales como artificiales solo pueden modificar a un objeto geográfico, pero no iniciarlo o finalizarlo. Esto es, los objetos geográficos existen siempre durante todo el tiempo en que se realiza la descripción de un entorno geográfico. Entonces, lo que causan los eventos es que modifican sus propiedades y relaciones espaciales con otros objetos geográficos.

En general, cada una de estas relaciones tiene un objetivo en particular.

Inicia se usa para indicar:

- Un fenómeno geográfico inicia un evento natural.

- Un objeto geográfico inicia un evento artificial.

- Un evento natural inicia un evento artificial.

- Un evento natural inicia un evento natural.

- Un evento artificial inicia un evento artificial.

Modifica se utiliza cuando:

- Un fenómeno geográfico modifica un evento natural.

- Un objeto geográfico modifica un evento artificial.

- Un evento natural modifica un evento natural.

- Un evento natural modifica un evento artificial.

- Un evento artificial modifica un evento artificial.

- Un evento artificial modifica un objeto geográfico.

- Un evento natural modifica un objeto geográfico.

Finaliza se usa en los siguientes casos:

- Un fenómeno geográfico finaliza un evento natural.

- Un objeto geográfico finaliza un evento artificial.

- Un evento natural finaliza un evento artificial.

- Un evento natural finaliza un evento natural.

- Un evento artificial finaliza un evento artificial.

Se puede observar que las relaciones inicia, modifica y finaliza son elementos del conjunto $\omega$, estas relaciones son los axiomas propuestos y suficientes para describir lo que ocurre en una conceptualización de un dominio geográfico, es decir, nos permiten relacionar temporal- mente la secuencia de los sucesos que ocurren en el entorno.

Entonces, cuando se forma la historia de una entidad geográfica por las cadenas de cambio y por estas relaciones, también está compuesta por las relaciones entre eventos.

\section{Relaciones entre historias}

En la ecuación (1) se realiza la descripción del entorno geográfico, indicada como la unión de las historias individuales de las entidades geográficas. La forma en que se realiza esta unión es por medio de las relaciones entre las historias. Entre dos historias pueden ocurrir los siguientes casos:

Sea $h_{i}$ y $h_{j} \in H$, donde $H$ es el conjunto de las historias que pertenecen a la descripción del entorno geográfico, entonces:

$h_{i} \cap h_{j}\left\{\begin{array}{l}=\varnothing \text { Disjoint } \\ \neq \varnothing \text { Intersect }\end{array}\right.$

La obtención de la medición cuantitativa es relativamente sencilla, pues sólo considera el tiempo en que dos historias se intersecan, más no la importancia de estas intersecciones. Supongamos que tenemos las historias $h_{i}$ y $h_{j}$, donde $i \neq j$. Es necesario definir la función $\operatorname{dur}($ ), la cual obtiene la duración, medida en unidades de tiempo, para una historia. La ecuación (5) define dicha función

$$
\begin{gathered}
\operatorname{dur}\left(h_{i}\right) \rightarrow R^{+} \\
h_{i} \in H
\end{gathered}
$$

Entonces, la relevancia que hay entre $h_{i}$ y $h_{j}$ es el grado en que dos historias están intersecadas. De esta forma obtenemos la medida cuantitativa en que se relacionan un par de historias. La función rel( ) obtiene esta medida y se define en la ecuación (6)

$$
\operatorname{rel}\left(h_{i}, h_{j}\right)=\frac{\sum_{k=1}^{n} \operatorname{dur}\left(h_{i} \cap_{k} h_{j}\right)}{\min \left[\operatorname{dur}\left(h_{i}\right), \operatorname{dur}\left(h_{j}\right)\right]}
$$

donde

$n$ es el número de veces que se intersectan $h_{i} \mathrm{y} h_{j}$

$k$ es el índice de cada una de estas intersecciones.

Esta medida puede ser de dos tipos: cuantitativa y cualitativa. En la figura 2 podemos ver una posible jerarquía con la cual podemos clasificar la intersección entre las 


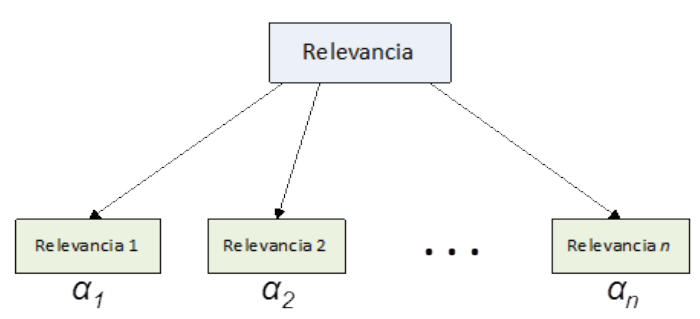

Figura 2. Jerarquía de relevancia

historias $h_{i}$ y $h_{j}$. Para poder calificar la relevancia de forma cualitativa de la intersección entre dos historias, podemos utilizar una jerarquía ordenada (Levachkine y Guzman, 2004).

También en la figura 2 se aprecia que para cada partición de relevancia se asigna un valor $\alpha_{i}$ que es el valor con el que podemos mapear el resultado obtenido de la función $\mathrm{rel}($ ) para poder calificar la intersección entre dos historias. Como los valores $\alpha_{i}$ son los límites de cada partición, para el caso de la jerarquía de la figura 2 tenemos (ecuación 7):

$0 \leq\left(\alpha_{i}<\alpha_{j}\right) \leq 1, \quad 1 \leq i \leq n, \quad 1 \leq j \leq n, \quad$ con $i \neq j$

donde $n$ es el número de particiones.

Existen varias formas de asignar estos valores $\alpha_{i}$ queda claro que esto depende en gran medida del contexto. Una forma de asignar estos valores puede ser directamente consultando algún experto. Otra manera de asignar estos valores puede ser usando medidas estadísticas o de alguna otra forma que sea útil para el contexto en que sean utilizados. También podemos ver el lugar donde se intersecan dos historias como los momentos donde sucede algún evento, el cual relaciona ambas historias.

Cabe mencionar que tanto las relaciones espaciales como las temporales se plantean como relaciones base, es decir, sirven para definir nuevas relaciones que sean más adecuadas semánticamente para los contextos sobre los cuales se desee trabajar. Por ejemplo, la relación topológica dentro puede utilizarse para definir una nueva relación llamada fuera de la siguiente forma: fuera $\rightarrow$ ! dentro. De esta menra es posible generar una ontología para conceptualizar estas nuevas relaciones basadas en las planteadas en este artículo.

\section{Algoritmo general}

En la sección anterior se explican las partes que conforman el modelo propuesto y lo que cada una de ellas trata de representar conceptualmente. En este punto se pre- senta un algoritmo que pretende usar los componentes del modelo y obtener como salida la esperada descripción explícita de un entorno geográfico dinámico.

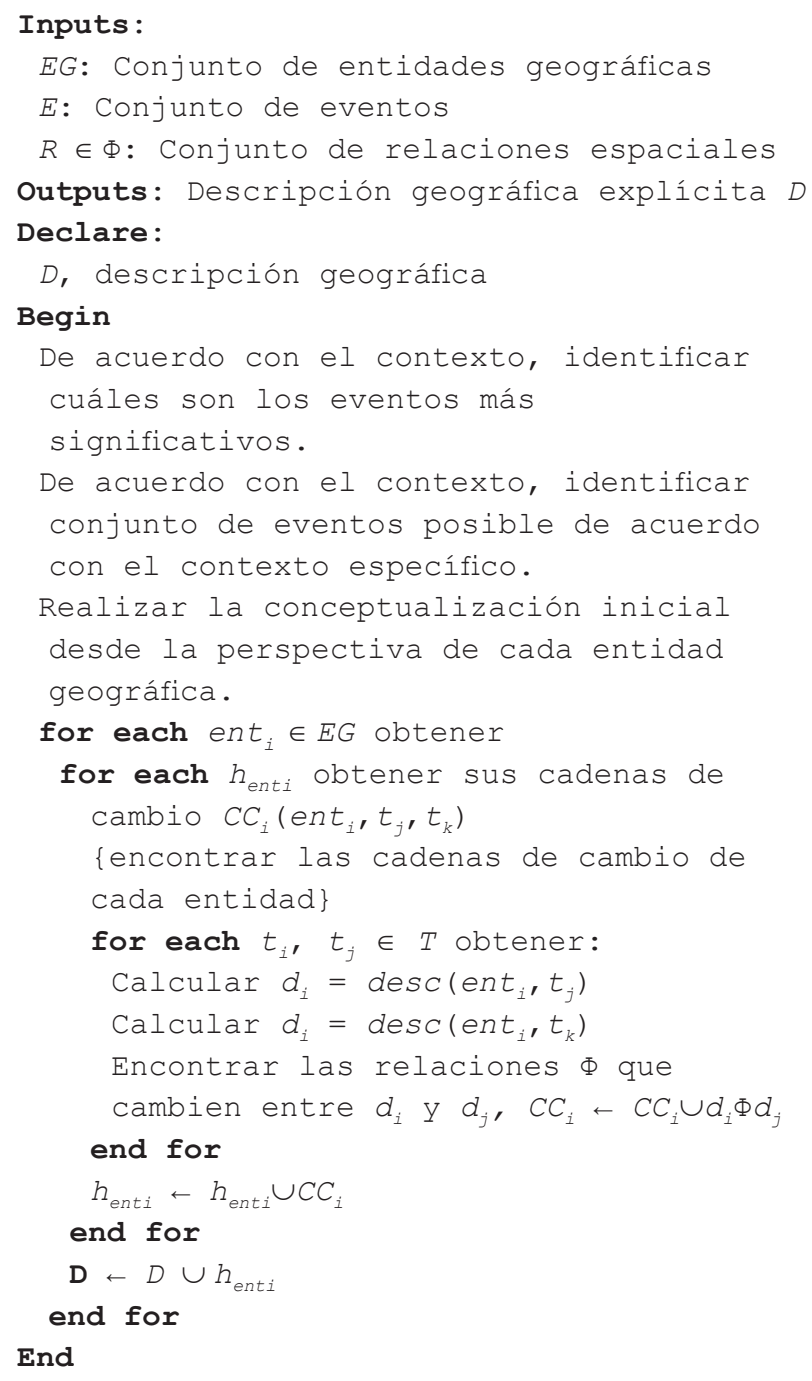

Algunas partes del algoritmo deben ejecutarlas algún ente externo, el cual puede ser una persona experta en el área de aplicación que se pretenda describir. Respecto al tipo de datos de entrada, queda abierto de acuerdo a la aplicación específica donde se desee implementar, puesto que al ser un modelo general la implementación depende en gran medida de considerar estos aspectos.

\section{Resultados}

En esta sección presentamos un ejemplo como caso de estudio para mostrar la utilidad del modelo propuesto. En este ejemplo tratamos el caso donde los principales 
objetos geográficos son dos personas, las cuales despiertan, salen de su casa, se transportan al trabajo y luego van de regreso a sus casas.

Siguiendo el algoritmo, lo primero es identificar cuáles son los objetos geográficos que participarán en la descripción. Para este caso los objetos que identificamos son: persona $A$, persona $B$, transporte 437 , transporte 734. Una vez identificadas las entidades geográficas, se prosigue a identificar los eventos posibles o permisibles para el propósito de la descripción. Así, los eventos que se identifican son: despierta, desayuna, subir al transporte, bajar del transporte, llegar al trabajo, salir del trabajo y entrar a casa.

Teniendo estos dos elementos se procede a obtener las cadenas de cambio para la persona $A$ y la persona $B$, basándose en las descripciones sucesivas de cada entidad geográfica. Estas cadenas quedan descritas en las tablas 3 y 4 .

Tabla 3. Descripciones persona A

$\operatorname{desc}_{0}(A, 6: 45)=\{$ dormido $\}$

$\operatorname{desc}_{1}(A, 7: 00)=\{$ despierto $\}$

$\operatorname{desc}_{2}(A, 8: 00)=\{$ dentro de cocina, desayunando $\}$

$\operatorname{desc}_{3}(A, 8: 30)=\{$ dentro de transporte-437\}

$\operatorname{desc}_{4}(A, A, 9: 00)=\{$ fuera transporte-437 $\}$

$\operatorname{desc}_{5}(A, 9: 15)=\{$ dentro trabajo $\}$

$\operatorname{desc}_{6}(A, 18: 00)=\{$ enfrente trabajo $\}$

$\operatorname{desc}_{7}(A, 18: 30)=\{$ dentro transporte- 734$\}$

$\operatorname{desc}_{8}(A, 19: 00)=\{$ fuera transporte-734\}

$\operatorname{desc}_{9}(A, 19: 30)=\{$ dentro casa $\}$

Tabla 4. Descripciones persona B

$\operatorname{desc}_{0}(\mathrm{~B}, 6: 45)=\{$ dormido $\}$

$\operatorname{desc}_{1}(\mathrm{~B}, 6: 50)=\{$ despierto $\}$

$\operatorname{desc}_{2}(\mathrm{~B}, 7: 30)=\{$ dentro cocina, desayunando $\}$

$\operatorname{desc}_{3}(\mathrm{~B}, 8: 30)=\{$ dentro transporte -437$\}$

$\operatorname{desc}_{4}(\mathrm{~B}, 8: 50)=\{$ fuera transporte -437$\}$

desc $_{5}(\mathrm{~B}, 9: 00)=\{$ dentro trabajo $\}$

$\operatorname{desc}_{6}(\mathrm{~B}, 18: 00)=\{$ enfrente trabajo $\}$

$\operatorname{desc}_{7}(\mathrm{~B}, 18: 30)=\{$ dentro transporte -734$\}$

$\operatorname{desc}_{8}(\mathrm{~B}, 19: 00)=\{$ fuera transporte-734\}

$\operatorname{desc}_{9}(\mathrm{~B}, 19: 25)=\{$ dentro casa $\}$

Tabla 5. Cadena de cambio de persona A

$C C_{1}(A)=\left\{\operatorname{desc}\left(A, t_{0}\right) \Phi \operatorname{desc}\left(A, t_{1}\right)\right\}=\{$ despierta $\}$

$C C_{2}(A)=\left\{\operatorname{desc}\left(A, t_{1}\right) \Phi \mathrm{d} \operatorname{desc}\left(A, t_{2}\right)\right\}=\{$ desayuna $\}$

$\operatorname{CC}_{3}(A)=\left\{\operatorname{desc}\left(A, t_{2}\right) \Phi \operatorname{desc}\left(A, t_{3}\right)\right\}=\{$ sube al transporte-437\}

$C_{4}(A)=\left\{\operatorname{desc}\left(A, t_{3}\right) \Phi \operatorname{desc}\left(A, t_{4}\right)\right\}=\{$ baja del transporte- 437$\}$

$\operatorname{CC}_{5}(A)=\left\{\operatorname{desc}\left(A, t_{4}\right) \Phi \operatorname{desc}\left(A, t_{5}\right)\right\}=\{$ entra al trabajo $\}$

$\operatorname{CC}_{6}(A)=\left\{\operatorname{desc}\left(A, t_{5}\right) \Phi \operatorname{desc}\left(A, t_{6}\right)\right\}=\{$ sale del trabajo $\}$

$\mathrm{CC}_{7}(A)=\left\{\operatorname{desc}\left(A, t_{6}\right) \Phi \operatorname{desc}\left(A, t_{7}\right)\right\}=\{$ sube al transporte- 734$\}$

$C C_{8}(A)=\left\{\operatorname{desc}\left(A, t_{7}\right) \Phi \operatorname{desc}\left(\mathrm{v}, t_{8}\right)\right\}=\{$ baja del transporte- 734$\}$

$C C_{9}(A)=\left\{\operatorname{desc}\left(A, t_{8}\right) \Phi \operatorname{desc}\left(A, t_{9}\right)\right\}=\{$ entra a casa $\}$
Tabla 6. Cadena de cambio de persona B

$C C_{1}(\mathrm{~B})=\left\{\operatorname{desc}\left(\mathrm{B}, t_{0}\right) \Phi \operatorname{desc}\left(\mathrm{B}, t_{1}\right)\right\}=\{$ despierta $\}$

$C_{2}(\mathrm{~B})=\left\{\operatorname{desc}\left(\mathrm{B}, t_{1}\right) \Phi \operatorname{desc}\left(\mathrm{B}, t_{2}\right)\right\}=\{$ desayuna $\}$

$C_{3}(\mathrm{~B})=\left\{\operatorname{desc}\left(\mathrm{B}, t_{2}\right) \Phi \operatorname{desc}\left(\mathrm{B}, t_{3}\right)\right\}=\{$ sube al transporte -437$\}$

$C C_{4}(\mathrm{~B})=\left\{\operatorname{desc}\left(\mathrm{B}, t_{3}\right) \Phi \operatorname{desc}\left(\mathrm{B}, t_{4}\right)\right\}=\{$ baja del transporte -437$\}$

$C C_{5}(\mathrm{~B})=\left\{\operatorname{desc}\left(\mathrm{B}, t_{4}\right) \Phi \operatorname{desc}\left(\mathrm{B}, t_{5}\right)\right\}=\{$ entra al trabajo $\}$

$C_{6}(\mathrm{~B})=\left\{\operatorname{desc}\left(\mathrm{B}, t_{5}\right) \Phi \operatorname{desc}\left(\mathrm{B}, t_{6}\right)\right\}=\{$ sale del trabajo $\}$

$C C_{7}(\mathrm{~B})=\left\{\operatorname{desc}\left(\mathrm{B}, t_{6}\right) \Phi \operatorname{desc}\left(\mathrm{B}, t_{7}\right)\right\}=\{$ sube al transporte -734$\}$

$C C_{8}(\mathrm{~B})=\left\{\operatorname{desc}\left(\mathrm{B}, t_{7}\right) \Phi \operatorname{desc}\left(\mathrm{B}, t_{8}\right)\right\}=\{$ baja del transporte- 734$\}$

$C_{9}(\mathrm{~B})=\left\{\operatorname{desc}\left(\mathrm{B}, t_{8}\right) \Phi \operatorname{desc}\left(\mathrm{B}, t_{9}\right)\right\}=\{$ entra a casa $\}$

En la tabla 5 se obtienen los valores para el operador $\Phi$. Como se puede observar en $C C_{1}(A)$ donde $\Phi=$ despierta, lo que describe el cambio ocurrido entre las dos descripciones. Este cambio debe conceptualizarse previamente de acuerdo con el contexto que se esté trabajando. Para este caso, el cambio en la propiedad de la entidad puede describirse como: despierta $=$ dormido $\rightarrow$ despierto .

Esta conceptualización debe hacerse para una de las relaciones que se deseen utilizar para describir los cambios que lleguen a ocurrir.

Con las cadenas de cambio generadas podemos formar las historias para cada una de las entidades geográficas. De este modo las historias quedan formadas de la siguiente manera:

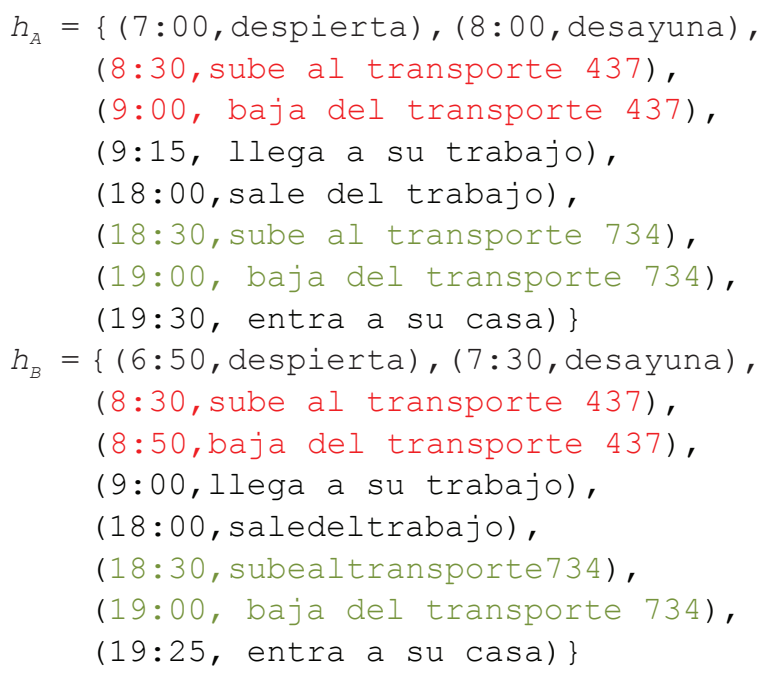

La descripción final resulta de la unión de estas dos historias de acuerdo con lo explicado sobre la unión de las historias. La clave es encontrar el punto en que éstas se relacionan, pues es lo que nos proporciona información adicional que con modelados tradicionales no se obtendría. En este ejemplo, si representamos las líneas de tiempo de las historias que se verían como se muestra en la figura 3. 

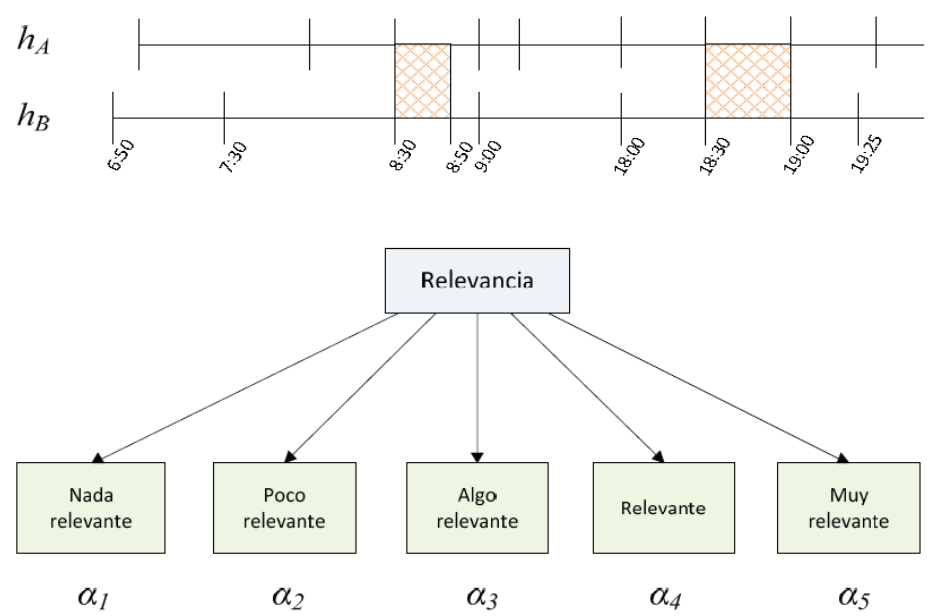

Figura 3. Líneas de tiempo e intersección entre estas

Figura 4. Medida de relevancia jerarquizada
En la figura 3 podemos observar que hay dos intersecciones entre las historias $h_{A}$ y $h_{B}$. Esta intersección está dada por $h_{A} \cap h_{B}=\{[8: 30,8: 50],[18: 40,19: 00]\}$ y como se ve es diferente de $\Phi$. Partiendo de este hecho podemos pensar en una forma de medir el grado en que dos historias están relacionadas. Esta medida puede ser dos tipos: cuantitativa y cualitativa. En la figura 4 se puede ver una posible jerarquía con la que podemos clasificar la intersección entre las historias $h_{A}$ y $h_{B}$.

Para este ejemplo podemos asignar los valores considerando una separación equidistante entre cada nodo, entonces se tienen los siguientes valores: $\alpha_{1}=0, \alpha_{2}=0.25$, $\alpha_{3}=0.50, \alpha_{4}=0.75, \alpha_{5}=1.0$. Como la evaluación de la función rel( ) no siempre arroja un valor igual a algún $\alpha_{i^{\prime}}$ podemos asignar intervalos disjuntos de la siguiente forma:

$\alpha_{1}=0, \alpha_{2}=(0,0.25], \alpha_{3}=(0.25,0.50], \alpha_{4}=(0.50,0.75], \alpha_{2}=(0.75,1]$

De estos intervalos, se observa que todos son abiertos por la izquierda y cerrados por la derecha, esto con el fin de tener intervalos disjuntos. De esta forma es fácil decidir cómo clasificar la relevancia entre la intersección de dos historias. También es claro que esta definición puede llegar a ser muy tajante, por el hecho que los límites entre dos medidas de relevancia pueden no ser muy claros.

Utilizando la ecuación (6), se calcula la relevancia entre las historias.

$$
\operatorname{rel}\left(h_{A}, h_{b}\right)=\frac{20+20}{\min [765,760]}=\frac{40}{760}=0.053
$$

De acuerdo con estos valores, se puede decir que las historias son poco relevantes entre sí.

Cabe señalar que los resultados aquí presentados son preliminares, ya que aún se continúa desarrollando y extendiendo el modelo que aquí se propone. Sin em- bargo, el modelo y las ideas presentadas son una base fundamental para el desarrollo de un modelo más general que sirva para cualquier propósito. En la sección de conclusiones se describen trabajos a futuro que deben desarrollarse para lograr llegar al objetivo de poder representar explícitamente el tiempo en un sistema de información geográfica sin importar su naturaleza. Entre ellos, se destaca considerar más relaciones y más operadores con los cuales relacionar a los entes participantes en la descripción, así como el desarrollo de un álgebra de eventos para poder realizar consultas sobre la información generada.

Actualmente se está trabajando en un sistema que emplea este modelo para buscar información espacial y temporal con respecto a horarios, salas disponibles, eventos, así como la descripción del personal de una unidad académica con el fin de analizar el comportamiento del personal y el uso adecuado de las instalaciones.

\section{Conclusiones y discusión}

La complejidad de intentar incorporar el "tiempo" en los sistemas de información geográfica tiene muchos aspectos a considerar. Entre estos aspectos se encuentran los medios por los cuales se introduce la dinámica geográfica. Entre ellos tenemos los que se denominan eventos, los cuales se introducen en los sistemas GIS por medio de cambios y movimiento de los objetos geográficos.

Cabe recordar que el objetivo de este trabajo es presentar los conceptos generales para procesar información espacial y temporal en sistemas de información geográfica, quedando como desarrollo su implementación. Asimismo que la definición de nuevas relaciones y conceptos dependerán del contexto en el que se trabaja. Esta conceptualización puede realizarse con el uso de ontologías, ya que por medio de esta herramienta es po- 
sible procesar este tipo de representación conceptual. También resalta el objetivo de llevar este modelo hacia el desarrollo de nuevas aplicaciones que procesen datos en tiempo real como los sistemas de monitoreo y advertencia, los cuales, con ayuda de estas nuevas conceptualizaciones, la calidad y la rapidez de las respuestas, serán más significativas.

Los diferentes tipos de eventos, además de ser dependientes de la escala, tienen diferencias, las cuales solo pueden determinarse por la situación en la cual aparecen o se utilizan. Muchas de estas múltiples definiciones dependen de la perspectiva. Nuevos desarrollos en análisis de video y algoritmos de rastreo pueden proveer nuevos métodos para identificar el movimiento en series cronológicas de datos. Sin embargo, el manejo efectivo de división y fusión de objetos geográficos sigue siendo un reto para la investigación en cuanto a entender la dinámica geográfica.

De acuerdo con lo escrito, los enfoques con los que se ha tratado de ligar el problema son igual de variados. Esto nos lleva a distintas formas de atacar el problema, pero ninguno de los enfoques ha logrado desambiguar las diferencias y similitudes entre los eventos, ni en sus observadores (cambio y movimiento). Con este trabajo se hace un intento en este sentido de desambiguar estos conceptos al tratar a todos de igual forma.

Uno de los trabajos a futuro es que al generar las historias por cada entidad se enriquece el contenido semántico de las descripciones de cada una de las entidades, la cantidad de información que debe procesarse es mayor, por lo cual se deben desarrollar métodos y algoritmos dedicados al procesamiento de esta información.

En esta parte surge el problema de cómo identificar qué entidades deben relacionarse con la entidad geográfica a describir, debido a que si utilizamos la respuesta más sencilla, es decir, si se relaciona con todas las otras entidades, el resultado va a ser que el cómputo requerido para realizar ese proceso será muy grande (si hay $n$ entidades, se realizarían $n^{2}$ descripciones por cada instante en que se desee realizar una descripción), ya que se estarían considerando relaciones con entidades que no sería necesario relacionar. La manera en que se puede trabajar con este problema es utilizar el contexto general del entorno.

Para ser más específicos, se propone un contexto local de interés (CLI), el cual es, a groso modo, un contexto reducido derivado del contexto del entorno general, especializado para agrupar pequeños conjuntos de entidades y eventos afines a la entidad geográfica de interés para, de esta forma, reducir el número de descripciones generadas. También es necesario realizar la formalización del CLI para después desarrollar programas que lo realicen en forma automática.
Respecto a la medida de relevancia entre pares de historias, esta puede modificarse para poder ser más explícitos al decir cuál es la relevancia de una historia con respecto a otra. Por ejemplo, supongamos que dos historias se intersecarán por la mitad de su duración total, esto no significaría que por ese hecho sean relevantes. Por otro lado, si esas mismas dos historias se intersecan por un periodo muy pequeño, no necesariamente significaría que no son relevantes entre sí. Es decir, se necesitan considerar más aspectos y no solo la cantidad de tiempo que se intersecan. Por esta razón se propone agregar algún modificador que pueda hacer que algunas intersecciones tengan más peso con respecto a otras considerando en qué aspectos se están relacionando, para poder medir cualitativamente la relevancia entre dos historias.

Trabajos adicionales indican que, con base en el modelo propuesto, se desarrollen motores de inferencia que puedan tener la capacidad de generar nuevo conocimiento a partir de las descripciones obtenidas, con el fin de generar un lenguaje enfocado exclusivamente al manejo de eventos en entornos geográficos dinámicos.

Otro reto, y trabajo futuro muy importante, es poder estandarizar la manera en que deben utilizarse los medios y los observadores para poder generar las descripciones de forma automática, sin importar el origen de los datos o su forma de representación para que el modelo pueda funcionar adecuadamente.

Es claro que aún quedan muchos trabajos por desarrollar, pero lo que se presenta en este documento brinda una buena base para iniciar y continuar con la extensa investigación en esta área del conocimiento.

\section{Referencias}

Campos J., Hornsby K. Temporal Constraints Between Cyclic Geographic Events, Proceedings of Geo Info, Campos do Jordao, Brazil, noviembre 2004, pp. 22-24.

Chang SK., Jungert E., Li Y. The Design of Pictorial Databases Based Upon the Theory of Symbolic Projections, Buchmann A., Günther-O. Smith T. y Wang Y., editores, en: Symposium on the Design and Implementation of Large Spatial Databases, LNCS, vol. 409, Nueva York, Springer-Verlag, 1989, pp. 303-323.

Chrisman N. Beyond the Snapshot: Changing the Approach to Change, Error and Process, en: Spatial and Temporal Reasoning in Geographic Information Systems, en: Egenhofer M. y Golledge R., editores, Spatial Information Systems, Nueva York, Oxford University Press, 1998, pp. 85-93.

DiBiase D., MacEachren A., Krygier J., Reeves C. Animation and the Role of Map Design in Scientific Visualization. Cartography and Geographic Information System, volumen 19 (número 4) 1992, pp. 201-214. 
Egenhofer M.F. A Formal Definition of Binary Topological Relationships, Third International Conference on Foundations of Data Organization and Algorithms (FODO), París, Francia, editores Litwin W., Schek H., LNCS, vol. 367, Springer-Verlag, junio 1989, pp. 457-472.

Egenhofer M.F., Herring J. Categorizing Binary Topological Relations Between Regions, Lines, and Points in Geographic Databases, Technical Report, Department of Surveying Engineering, University of Maine, 1990.

Freeman J. The Modeling of Spatial Relations. Computer Graphics and Image Processing, volumen 4 (número 2) 1975: 156-171.

Galton A. Fields and Objects in Space, Time and Space-time. Spatial Cognition and Computation, volumen 4 (número 1), 2004: 39-68.

Galton A. y Worboys M.F. Processes and Event in Dynamic Geospatial Networks, en: Proceedings of First International Conference on Geospatial Semantics, LNCS, vol. 3799, Springer 2004, pp. 45-59.

Hales S.D. y Johnson T.A. Endurantism, Perdurantism and Special Relativity. The Philosophical Quarterly, volumen 53 (número 213), 2003: 524-539.

Hernandez D. Relative Representation of Spatial Knowledge: The 2-D Case, en: Mark D. y Frank A. editores, Cognitive and Linguistic Aspects of Geographic Space, Kluwer Academic Publishers, Dordrecht, 1991.

Hornsby K. y Egenhofer M. Identity-Based Change: a Foundation for Spatio-Temporal Knowledge Representation. International Journal of Geographical Information Science, volumen 14 (número 3), 2000: 207-224.

Hornsby K. y Cole S. Modeling Moving Geospatial Objects from an Event-based Perspective, Transactions in GIS, volumen 11 (número 4), 2007: 555-573.

Kainz W. Order, Topology and, Metric in GIS, en: ASPRS-ACSM, Annual Convention. Baltimore, MD, marzo 1989, pp-154-160.

Langran G. Time in Geographical Information Systems, Londores, Taylor and Francis, 1972.

Levachkine S. y Guzman-Arenas A. Hierarchies Measuring Qualitative Variables, LNCS, vol. 2945, Springer-Verlag, 2004, pp. 258-270.

Martínez-Rosales M. Descriptor topológico para mapas topográficos, tesis (Maestría), Centro de Investigación en Computación, 2006.
Peuquet D.J. Representations of Space and Time, Neueva York, Guilford, 2002.

Peuquet D.J. Making Space for Time: Issues in Space-Time Data Representation, GeoInformatica, volumen 5 (número 1), 2001: 11-32.

Peuquet D.J. y Wentz E. An Approach for Time-Based Analysis of Spatiotemporal Data, en: Proceedings of Sixth International Symposiumof Spatial Data Handling, SDH ‘94, Waugh T.C. y Healey R.G. editores, Edinburgh, Scotland, 1994, pp. 489-504.

Peuquet D.J. It's Aabout Time: A Conceptual Framework for the Representation of Temporal Dynamics in Geographic Information Systems. Annals of the Association of American Geographers, volumen 84 (número 3), 1994: 441-46.

Peuquet D.J. y Ci-Xiang Z. An Algorithm to Determine the Directional Relationship Between Arbitrarily-Shaped Polygons in the Plane. Pattern Recognition, Endnote XML BibTex, volumen 20 (número 1), 1987: 65-74.

Raper J. Multidimensional Geographic Information Science, Londres y Nueva York, Taylor and Francis, 2000.

Worboys M.F. Unifying the Spatial and Temporal Components of Geographic Information, en: Waugh T.C. y Healey R.G. editores, Advances in Geographic Information Systems: Proceedings of the Sixth International Symposium on Spatial Data Handling, Edinburgh. Londres, Taylor and Francis, 1994, pp. 505-17.

Worboys M. y Hornsby K. From Objects to Events: GEM, the Geospatial Event Model, en: Egenhofer M., Freksa C., Miller H. editores, Proceeding of GIScience, Lecture Notes in Computer Science, 3234, Springer, Berlin, 2004, pp. 327-343.

\section{Este artículo se cita: \\ Citación estilo Chicago}

Martínez-Rosales, Miguel Alejandro, Levachkine, Serguei. Modelo conceptual de entornos geográficos dinámicos. Ingeniería Investigación y Tecnología, XV, 02 (2014): 163-174.

\section{Citación estilo ISO 690}

Martínez-Rosales M.A., Levachkine S. Modelo conceptual de entornos geográficos dinámicos. Ingeniería Investigación y Tecnología, volumen XV (número 2), abril-junio 2014: 163-174.

\section{Semblanza de los autores}

Miguel Alejandro Martínez-Rosales. Es estudiante de posgrado del programa de doctorado en ciencias de la computación del Centro de Investigación en Computación del IPN. Realizó sus estudios como ingeniero en sistemas computacionales durante el periodo de 1999-2003. Obtuvo el grado de maestro en ciencias de la computación en el año 2006, por el Centro de Investigación en Computación, IPN. Sus áreas de interés son el uso de ontologías temporales, descripción espacio-temporal de eventos.

Serguei Levachkine. Actualmente es profesor investigador del Centro de Investigación en Computación del Instituto Politécnico Nacional. Ha sido autor y co-autor de más de 150 publicaciones en congresos y revistas. Es egresado de la Universidad Estatal de Moscú (Lomonosov). Entre sus áreas de interés están el desarrollo de GIS (cartografía digital inteligente, métodos de representación del conocimiento espacial) e inteligencia artificial (procesamiento semántico de datos digitales, ontologías y sistemas basados en conocimiento). 